PROCEEDINGS OF THE

AMERICAN MATHEMATICAL SOCIETY

Volume 130, Number 11, Pages 3219-3230

S 0002-9939(02)06698-4

Article electronically published on May 29, 2002

\title{
ON DYNAMICS OF VERTICES OF LOCALLY CONNECTED POLYNOMIAL JULIA SETS
}

\author{
A. BLOKH AND G. LEVIN
}

(Communicated by Michael Handel)

\begin{abstract}
Let $P$ be a polynomial whose Julia set $J$ is locally connected. Then a non-preperiodic non-precritical vertex of $J$ must have the limit set which coincides with the limit set of an appropriately chosen recurrent critical point of $P$. In particular, if all critical points of $P$ are non-recurrent then all vertices of $J$ are preperiodic or precritical.
\end{abstract}

\section{INTRODUCTION}

In this paper $P$ denotes a complex polynomial with the Julia set $J$. Properties of $P \mid J$ and topological properties of $J$ have been studied in holomorphic dynamics for quite some time (see e.g. [BH], [DH], [Do, [F], [H], [J], [McM], [Mi], Sul], Th]). From now on we assume in this paper that $J$ is locally connected and we study the orbits of the vertices of $J$. As a corollary we prove that if all critical points of $P$ are non-recurrent, then all vertices of $J$ are preperiodic or precritical.

It is known that if $J$ is locally connected, then it is connected. By a Caratheodory theorem [CL, any point $x \in J$ is accessible from the basin of attraction of infinity $A_{\infty}$ by one or several paths tending to $x$ which can be chosen to be so-called external rays $[\mathrm{DH}]$. Let $N(x)$ be the number of such rays, and call points $x$ with $N(x) \geq 3$ vertices of $J$ (one can also define $N(x)$ as the number of components of the set $J \backslash\{x\}$ ). In the language of continuum theory, vertices are cut points cutting the Julia set in at least three components. The following problem is fundamental for understanding the topology of Julia sets; it was posed in the framework of polynomial laminations and solved for quadratic laminations in Th.

Problem 1. Is it true that all vertices of the locally connected Julia set are either precritical or preperiodic?

If we think of $J$ as a "graph" with, perhaps, infinitely many vertices, then it is natural to expect that in some cases $P$ acts on $J$ similarly to the way it would act on finite "graphs". Now, if a finite "graph" $G$ is invariant for $P$, then all vertices of $G$ are precritical or preperiodic (a point is preperiodic - resp. precritical - if it is mapped onto a periodic - resp. critical - point by $f^{k}, k \geq 0$ ). This makes the question above rather natural.

Received by the editors December 22, 2000.

2000 Mathematics Subject Classification. Primary 37F10; Secondary 37E25.

Key words and phrases. Julia set, vertices, laminations, recurrent critical points.

The first author was partially supported by NSF grant DMS 9970363. 
Related questions in the degree higher than 2 were considered by Kiwi Ki] who proved that for a polynomial of degree $d$ the number $N(x)$ of rays landing at a non-preperiodic non-precritical point $x \in J$ is at most $d$ (this is another proof of the result of [Th] in the quadratic case). In BL1, BL2] we obtained further results one of which is described in Theorem 2; it is easy to see, that it implies the results mentioned above.

By the grand orbit ( of $x$ ) we mean the union of all preimages of all iterates of $x$. Note that the number $N\left(x^{\prime}\right)$ is the same for all points $x^{\prime}$ of the grand orbit of $x$ if this orbit contains no critical points. By a Siegel disc we understand a Fatou component whose boundary is a simple closed curve such that an appropriate power of the map $P$ on its boundary is an irrational rotation.

Theorem 2 ([BL1], BL2]). $\sum_{x \in \Gamma}(N(x)-2) \leq k_{\sim}-k_{S}-1 \leq d-2$, where $\Gamma$ is a non-empty collection of non-preperiodic non-precritical points $x \in J$ from distinct grand orbits with $N(x) \geq 3, k_{\sim}$ is the number of distinct grand orbits of nonpreperiodic critical points $c \in J(P)$ with $N(P(c))=1$ (i.e., $P(c)$ is the landing point of exactly one external ray) and $k_{S}$ is the number of periodic orbits of the Siegel discs.

In fact, a complete analog of Theorem 2 is proven in [BL1, BL2 for invariant laminations and their factors which implies (as a particular case) Theorem 2 for polynomials with locally connected Julia sets.

In a way, Theorem 2 already shows to what extent the dynamics of a polynomial on its locally connected Julia set is similar to the dynamics of a polynomial on a finite graph: even if non-preperiodic non-precritical vertices exist, they form at most $d-2$ grand orbits. Observe, that although in general Theorem 2 does not solve Problem 1 and can only be considered as a step on the way to its solution, in some particular cases it implies that there are no non-preperiodic non-precritical vertices of a locally connected Julia set. In particular, Theorem 2 implies the following corollary.

Corollary 3 (cf. [Do, $[\mathrm{Po}]$ ). Let $P$ be a polynomial whose critical points are attracted by a periodic orbit or preperiodic. Then all vertices of $J$ are preperiodic or precritical.

In the present paper we suggest another class of polynomials such that all nonprecritical vertices of their Julia sets are preperiodic. A polynomial $P$ is called semi-hyperbolic [CJY] if no critical point of $P$ in $J$ is recurrent and there are no parabolic cycles. As follows from C.JY, the Julia set of such a polynomial is locally connected if it is connected, and hence every $x \in J$ is the landing point of finitely many external rays ([Ki], BL1], [BL2]). Assume, in addition, that $P$ has only repelling periodic points (and so its Julia set is a dendrite, i.e. locally connected tree-like continuum). Then using Yoccoz puzzle structure for $P$ we prove that all vertices of $J$ are preperiodic or precritical (we briefly outline the proof in Section 2 ). In fact, the same result holds for a wider class $\mathcal{A}$ of all polynomials with no recurrent critical points and with locally connected Julia set. Thus, compared to the above case in $\mathcal{A}$ we allow attracting or parabolic cycles.

If $J$ is a locally connected Julia set, then $x \in J$ is a local vertex of $J$ if there is a triod $Y \subset J$ whose branch point is $x$. If $J$ is a dendrite, this is equivalent to the usual definition, yet if there are bounded Fatou domains, the set of local vertices may be wider. 
Main Theorem. If $P \in \mathcal{A}$, then every local vertex of $J$ is either preperiodic or precritical.

A complication for $P \in \mathcal{A}$ is that $J$ no longer is a dendrite; we deal with it by developing topological dynamics of piecewise local homeomorphisms of unshielded plane continua which are backward stable (see definitions in Section 4), more precisely by describing the limit sets of local vertices of such maps. This description, applied to laminations and polynomials, yields Theorem 13 below which in turns implies the Main Theorem.

Theorem 13. Suppose that one of the assumptions below holds:

(1) $\sim$ is an invariant lamination and $f: J \rightarrow J$ is the corresponding factor map;

(2) $f$ is a polynomial, $J$ is its Julia set, and $J$ is locally connected.

Then if $x$ is a non-preperiodic non-precritical local vertex of $J$, then $\omega(x)$ coincides with $\omega(c(x))$ for a recurrent critical point $c(x)$ depending on $x$.

The paper is organized as follows. In Section 2 we outline the proof of the Main Theorem for semi-hyperbolic polynomials. In Section 3 we introduce the notion of unshielded continua and study their topology (connected Julia sets are unshielded continua). In Section 4 we study backward stable continuous maps of unshielded continua which are local homeomorphisms outside a finite set and prove Theorem 13.

We want to thank L. Oversteegen for useful discussions of the proofs of Lemmas 5 and 6 and to M. Misiurewicz for valuable discussions of the proof of Theorem 11. Also, we want to thank the referee for finding a gap in the original proof of the Main Theorem and one of the lemmas and for making valuable remarks which helped us improve the style of this paper.

\section{SEmi-Hyperbolic POLYNOMials Without ATtRACTING CYCLES}

In this section we consider a special case of semi-hyperbolic polynomials with all periodic points repelling, and outline the idea of proving the Main Theorem in this case.

Let $P$ be a polynomial of degree $d>1$ whose Julia set $J$ is connected and periodic points repelling. Assume that $P$ is a semi-hyperbolic polynomial [CJY] (i.e. every critical point in $J$ is not recurrent). By [JY], the Julia set of such a polynomial is locally connected, and hence, every $x \in J$ is a landing point of a finitely many external rays.

To demonstrate the idea of the proof in this case, consider Yoccoz puzzle structure for $P$. Note that in our case $P$ is not renormalizable, and the puzzle structure is constructed in a standard way (see e.g. [H], BL1]). Recall that for every $n=0,1, \ldots$ there are finitely many pairwise disjoint open puzzle pieces $Y_{n}^{1}, \ldots, Y_{n}^{k_{n}}$ of the level $n$. For any $Y=Y_{n}^{i}$, the intersection of the boundary $\partial Y$ of $Y$ with the Julia set has finitely many points (preperiodic by $P$ ). The union of the closures of $Y_{n}^{i}, 1 \leq i \leq k_{n}$, contains the Julia set $J$, and $P$ maps any puzzle piece of the level $n>0$ onto a puzzle piece of the level $n-1$.

Given a point $z \in J$, let $Y_{n}(z)$ be a nested sequence of open puzzle pieces, such that $z \in \overline{Y_{n}(z)}$ for any $n$. In [L], [BL1] we show that the Julia set $J$ is locally connected if and only if the intersection of all $Y_{n}(z)$ is the single point $z$, for each $z \in J$. 
Given a puzzle piece $Y=Y_{n}(z)$, we define a finite tree $T_{n}(z)$ as follows. As we know, the intersection of the boundary $\partial Y$ of $Y$ with the Julia set consists of finitely many points $x_{1}, \ldots, x_{p}$. Given any two of them $x_{i}, x_{j}$, let $\left[x_{i}, x_{j}\right]$ be the unique arc in $J$ with the ends $x_{i}, x_{j}$ (such an arc exists because $J$ is locally connected). Define now $T_{n}(z)$ as the union of all possible $\operatorname{arcs}\left[x_{i}, x_{j}\right]$. It is easy to see that $T_{n}(z)$ is a finite tree.

Then we use the fact that every critical point is non-recurrent and rely on a result of [BM] to find a number $m$ and a sequence $n_{k}$ such that the map $P^{n_{k}}$ maps homeomorphically the piece $Y_{m+n_{k}}(z)$ onto a piece of a fixed level $m$. Since there are finitely many pieces on each level, one can assume that $P^{n_{k}}\left(Y_{m+n_{k}}(z)\right)$ is a fixed piece $Y_{m}$.

Let us now assume that $z$ is neither preperiodic nor precritical, and then prove that $z$ is the landing point of at most 2 external rays. Assume (by contradiction) that there are at least 3 external rays landing at $z$. Then, for every $n$ large enough, $z$ is a vertex of every tree $T_{n}(z)$. From the above, $P^{n_{k}}$ maps $T_{n_{k}+m}(z)$ homeomorphically onto some tree $t_{k}$, and $z_{k}=P^{n_{k}}(z)$ is a vertex of $t_{k}$. By the construction, $t_{k}$ is a subset of $J$, and each end point of $t_{k}$ is a point of the set $\partial Y_{m} \cap J$. Since the latter set is finite, and $Y_{m}$ is a fixed piece, there are finitely many trees $t_{k}$, each of which is finite. Therefore, the points $z_{k}$ belong to a finite set of the vertices of the trees $t_{k}$. Thus $z_{k}=z_{i}$ for different $k, i$, which means that the point $z$ is preperiodic.

\section{TOPOLOGICAL PROPERTIES \\ OF LOCALLY CONNECTED UNSHIELDED CONTINUA}

A plane continuum $K$ is said to be unshielded [BO] if it coincides with the boundary of a domain $U$ which is a connected component of the complement of $K$. Connected Julia sets are unshielded since they coincide with the boundary of $A_{\infty}$. In this section we study topological properties of locally connected unshielded continua.

It immediately follows from the definition that an unshielded continuum $K$ cannot contain a continuum of the shape of the letter $\theta$. Therefore, the following so-called $\theta$-curve Theorem applies to unshielded continua.

Theorem 4 ( $\theta$-curve Theorem $[\mathrm{Kur})$. A locally connected unshielded continuum is hereditarily locally connected: all its subcontinua are locally connected. Also, any connected subset of a locally connected unshielded continuum is arcwise connected.

We need some standard definitions. An arc is a homeomorphic image of the interval $[0,1]$. If the endpoints of an arc are $a, b$ and if it causes no confusion we use the notation $[a, b]$.

The following lemma is proven in $[\mathrm{BO}$ and is given here for the sake of completeness.

Lemma $5([\mathrm{BO}])$. Let $T$ be a locally connected unshielded continuum. For any sequence of continua $M_{n} \subset J$ such that diameters of sets $M_{n}$ do not converge to 0 there exists a subsequence $K_{n}^{\prime}$ such that all sets $K_{n}^{\prime}$ contain some arc $L^{\prime}$.

Proof. We can always find a subsequence of continua $M_{n}$ converging in the Hausdorff sense to a non-degenerate continuum. By Theorem 4 all $M_{n}$ are locally connected and therefore arcwise connected. Choose subarcs $K_{n}$ of $M_{n}$ which contain points $u_{n}, v_{n} \in K_{n}$ positively distant from each other. We can assume that there are points $x \neq y$ such that $u_{n} \rightarrow x, v_{n} \rightarrow y$. Assuming that $u_{n}$ and $v_{n}$ are very 
close to $x, y$ respectively, and using the fact that $T$ is locally connected, we can find small disjoint $\operatorname{arcs} L_{n}^{\prime}=\left[x, u_{n}\right] \subset T, R_{n}^{\prime}=\left[y, v_{n}\right] \subset T$ so that $\lim L_{n}^{\prime}=\{x\}$ and $\lim R_{n}^{\prime}=\{y\}$.

Since $K_{n}$ is arcwise connected by Theorem 4, we can always find an arc $Q_{n}$ connecting $u_{n}$ and $v_{n}$ inside $K_{n}$. Then we can always choose points $x_{n} \in Q_{n} \cap L_{n}^{\prime}$ and $y_{n} \in R_{n}^{\prime} \cap Q_{n}$ so that the subarc $\left[x_{n}, y_{n}\right]$ of $Q_{n}$ connecting $x_{n}$ and $y_{n}$ inside $Q_{n}$ is disjoint from $L_{n}^{\prime} \cup R_{n}^{\prime}$ outside its endpoints $x_{n}, y_{n}$. Let $L_{n}=\left[x, x_{n}\right] \subset L_{n}^{\prime}$ $\left(R_{n}=\left[y, y_{n}\right] \subset R_{n}^{\prime}\right)$ be the arcs connecting $x$ and $x_{n}\left(y\right.$ and $\left.y_{n}\right)$ inside $L_{n}^{\prime}\left(R_{n}^{\prime}\right)$. Then $J_{n}=\left[x, x_{n}\right] \cup\left[x_{n}, y_{n}\right] \cup\left[y_{n}, y\right]$ is an $\operatorname{arc}, \lim L_{n}=\{x\}$ and $\lim R_{n}=\{y\}$. We can choose a subsequence now so that $\left[x_{n}, y_{n}\right]$ converge to the limit continuum $K$; observe that since $L_{n}, R_{n}$ converge to points, the arcs $J_{n}$ converge to the same continuum $K$. Let $X^{\prime}=K \cup_{n} J_{n}$, then $X^{\prime} \subset T$ is a continuum.

Choose an arc $A^{\prime}=[x, y] \subset K$ connecting $x$ and $y$. Then $\left[x, x_{n}\right] \cup\left[x_{n}, y_{n}\right] \cup\left[y_{n}, y\right] \cup$ $[y, x]=E_{n}$ is a closed curve for every $n$. Since $L_{n}$ and $R_{n}$ are very small for big $n$, we may assume that there exists a non-degenerate arc $A \subset A^{\prime} \backslash \bigcup_{n}\left[L_{n} \cup R_{n}\right]$. If $A \subset J_{n}$ for all $n$, then $A \subset\left[x_{n}, y_{n}\right] \subset K_{n}$ and we are done. Assume that there exists a point $a \in A \backslash J_{1}$. Then the fact that $E_{1}=\left[x, x_{1}\right] \cup\left[x_{1}, y_{1}\right] \cup\left[y_{1}, y\right] \cup[y, x]$ is a closed curve implies that there exists a bounded region $D$ complementary to $E_{1}$ such that $a$ is contained in $\partial D=S$. By Theorem $4 S$ is locally connected; since $S \subset T$ we see that $S$ is a simple closed curve. Then $A_{1}=S \cap A^{\prime}$ is a non-degenerate arc containing $a$ (both $S$ and $A^{\prime}$ are contained in an unshielded continuum $T$, and the intersection of an arc and a simple closed curve in an unshielded continuum is an arc or a point unless it is empty). Let $I_{1}=\overline{S \backslash A^{\prime}}$. Since $T$ contains no $\theta$-curve, any pair of distinct points $p, q \in S$ with $p \in I_{1}$ and $q \in A_{1}$ cuts each $J_{n}$ between $x$ and $y$. Hence either $A_{1}$ or $I_{1}$ is contained in infinitely many $J_{n}$. Since $L_{n} \rightarrow\{x\}, R_{n} \rightarrow\{y\}$ it is easy to find a non-degenerate arc $L^{\prime}$ which is contained in infinitely many $K_{n}$ as required.

Let us now introduce the following notion: a point $x$ of a hereditarily locally connected continuum $K$ is called a locally $\varepsilon$-essential vertex if there exists a triod $Y \subset K$ whose vertex is the point $x$ and whose branches are all of diameters at least $\varepsilon$ (in this paper we deal only with locally connected continua and thus give the definition in this particular case). A point which is a locally $\varepsilon$-essential vertex for some $\varepsilon>0$ is called a local vertex of $K$. Observe that a local vertex does not have to be a vertex (for example, if a continuum $T$ has the shape of a letter $\sigma$, then the only branching point of $T$ is a local vertex but not a vertex of $T$ ). Now, a point $x$ of a hereditarily locally connected continuum $X$ is said to be a globally $\varepsilon$-essential vertex if there are at least three components of $X \backslash\{x\}$ of diameter at least $\varepsilon$. Clearly, a globally $\varepsilon$-essential vertex is locally $\varepsilon$-essential, and all globally $\varepsilon$-essential vertices of $T$ taken over all $\varepsilon>0$ form the set of all vertices of $T$.

The following topological result is used in the proof of Theorem 10.

Lemma 6. Let $T$ be a locally connected unshielded continuum. Then for every $\varepsilon$ the number of locally $\varepsilon$-essential vertices of $T$ is finite (so in particular, the set of all local vertices is at most countable).

Proof. Suppose that there are infinitely many locally $\varepsilon$-essential vertices $\left\{a_{i}\right\}_{i=1}^{\infty}$. For every $i$ let $Y_{i}$ be a triod containing $a_{i}$ as its vertex and having branches $N_{i}, L_{i}$ and $M_{i}$ each of which has diameter greater than $\varepsilon$. Assume that $a_{i} \rightarrow a$. By Lemma 5 we can choose a subsequence and an arc $B$ so that $a \notin B, a_{i} \notin B$ for any $i$, and 
$B \subset N_{i}$ for any $i$ (indeed, we can always choose a subsequence and $B$ by Lemma 5 so that $a \notin B$ and then refine this subsequence so that $a_{n} \notin B$ for any $n$ ). Then we can refine this subsequence and choose another subsequence and an $\operatorname{arc} C$ so that $a \notin C, a_{i} \notin C$ for any $i$ and $C \subset L_{i}$ for any $i$; clearly then $C$ and $B$ are disjoint. Finally, we can refine the latest subsequence and find its subsequence and an arc $D$ so that $a \notin D, a_{i} \notin D$ for any $i$ and $D \subset M_{i}$ for any $i$; clearly then $C, B$ and $D$ are disjoint. Now, $Y_{1}$ contains $B, C$ and $D$ and so does $Y_{2}, Y_{3}, \ldots$ However, this is clearly impossible for the unshielded continuum $T$ to contain all these triods since in this case it is not unshielded, a contradiction.

\section{Main Theorem}

So far the arguments were purely topological; now it is the time to apply dynamical arguments and prove the Main Theorem. Our plan is to obtain some general results for a certain class of continuous maps of locally connected unshielded continua. Since polynomial maps on their locally connected Julia sets belong to this class of maps, we then apply our results and deduce Theorem 10, Theorem 13 and the Main Theorem.

First we need the following definition. A map $f: X \rightarrow X$ of a metric space $X$ into itself is said to be a local homeomorphism at a point $x$ if any small neighborhood of $x$ is homeomorphically mapped by $f$ onto a neighborhood of $f(x)$. A map is called a local homeomorphism on a set $Y \subset X$ if it is a local homeomorphism at every point of $Y$. We study local homeomorphisms of connected neighborhoods in a locally connected unshielded continuum below.

Lemma 7. Let $f: T \rightarrow T$ be a continuous map of a locally connected unshielded continuum $T$. Let $U \subset T$ be an open connected set and $V$ be a component of $f^{-1}(U)$ such that $f \mid V$ is a local homeomorphism. Then $V$ maps onto $U$ and, moreover, $f(\partial V)=\partial U$.

Proof. As a component of an open set in a locally connected space, $V$ is open. Since $f \mid V$ is a local homeomorphism, it is an open map, hence $f(V) \subset U$ is open. Let us show that $W=U \backslash f(V)$ is open too. Indeed, if $x \in W$ and yet no neighborhood of $x$ is inside $W$, then $x$ is the limit point of a sequence of points $f\left(y_{n}\right), y_{n} \in V$. Assume that $y_{n} \rightarrow y \in \partial V$ ( $y$ cannot belong to $V$ because $f(y) \notin f(V)$ ). Then $V \cup\{y\}$ is connected and maps into $U$, a contradiction with the choice of $V$. Hence, $W$ is open. But this together with the connectedness of $U$ implies that $W$ is empty and $f(V)=U$.

Let us show that $f(\partial V)=\partial U$. Indeed, otherwise one of the two cases must take place. First, there may exist a point $x \in \partial V$ which maps inside $U$. However, then $V \cup\{x\}$ is connected and maps into $U$, a contradiction with the choice of $V$. Second, if $y \in \partial U$, then because clearly $f(\bar{V})=\bar{U}$ we see that $y=f(z)$ for some $z \in \bar{V}$, and $z \in V$ is impossible since then $f(z)$ would belong to $U$. Thus, $z \in \partial V$ as desired.

The next lemma deals with local homeomorphisms of sufficiently small connected neighborhoods of local vertices.

Lemma 8. Let $U \subset T$ be a connected neighborhood of a local vertex $x$ in a locally connected unshielded continuum $T$ and $f: T \rightarrow T$ is a map such that $f \mid U$ is a local homeomorphism and $U$ is a component of $f^{-1}(f(U))$. Then the following hold: 
(1) for any simple closed curve $S \subset f(U)$ any component $R$ of its preimage inside $U$ is a simple closed curve such that $f(R)=S$;

(2) for any simple closed curve $R \subset U$ the set $f(R)$ is a simple closed curve also;

(3) the images of different components of $U \backslash\{x\}$ under a local homeomorphism $f: U \rightarrow T$ are disjoint.

Proof. (1) A set $R$ is a locally connected continuum since $T$ is hereditarily locally connected by Theorem 4 . Moreover, since $f$ is a local homeomorphism on $R$, then $R$ maps onto $S$ and has no endpoints or vertices, i.e. $R$ is a simple closed curve.

(2) Clearly, $f(R)$ contains a simple closed curve $S^{\prime}$, and so there exists a component $R^{\prime}$ of the preimage of $S^{\prime}$ having a non-trivial intersection with $R$. Since $T$ is unshielded, this implies that $R^{\prime}=R$ and $S^{\prime}=S$ as desired.

(3) Assume that there are components $Y_{1}, Y_{2}$ of $U \backslash\{x\}$ and points $y_{1} \in Y_{1}, y_{2} \in$ $Y_{2}$ with $f\left(y_{1}\right)=f\left(y_{2}\right)=z$. By Theorem 4 there exist $\operatorname{arcs}\left[x, y_{1}\right] \subset Y_{1}$ and $\left[x, y_{2}\right] \subset$ $Y_{2}$. Since $f$ is a homeomorphism on a small neighborhood of $x$ we may assume that there are points $z_{1} \in\left(x, y_{1}\right)$ and $z_{2} \in\left(x, y_{2}\right)$ such that $f\left(z_{1}\right)=f\left(z_{2}\right)$ and images of $\left(x, z_{1}\right)$ and $\left(x, z_{2}\right)$ are disjoint. Then $S=f\left[x, z_{1}\right] \cup f\left[x, z_{2}\right] \subset f(U)$ is a simple closed curve, so by (1) there exists a simple closed curve $R \supset\left[x, z_{1}\right] \cup\left[x, z_{2}\right]$ which contradicts the fact that $\left(x, z_{1}\right]$ and $\left(x, z_{2}\right]$ are contained in distinct components of $U \backslash\{x\}$.

If $f: T \rightarrow T$ is a local homeomorphism of a locally connected unshielded continuum $T$ everywhere but at a finite set $C_{f}$ of critical points, then we call it a piecewise local homeomorphism. Choose a metric $d(\cdot, \cdot)$ on $T$. For $x \in T$ and $n>0$ consider the family $\mathcal{B}(x, n)$ of all connected neighborhoods $U$ of $f^{n}(x)$ which can be pulled back to $x$ as local homeomorphisms. Thus, we include $U$ in $\mathcal{B}(x, n)$ if $U$ is a connected neighborhood of $f^{n}(x)$ and the component $V$ of $f^{-n}(U)$ which contains $x$ is such that $f^{n} \mid V$ is a local homeomorphism. Then the union of any collection of neighborhoods from $\mathcal{B}(x, n)$ belongs to the same family and there exists the maximal neighborhood $U_{n}(x)=U \in \mathcal{B}(x, n)$ and the corresponding neighborhood $V_{n}(x)=V$ of $x$. Denote the minimal distance $d\left(f^{n}(x), \partial U\right)$ between $f^{n}(x)$ and $\partial U$ by $r_{n}(x)=r_{n}$. Then $r_{n}>0$ if all points $x, \ldots, f^{n-1}(x)$ are not critical, otherwise define $r_{n}$ as 0 .

By the definition there are points $z$ on the boundary $\partial V$ of $V$ such that $f^{n}$ is not a local homeomorphism at $z$ and $d\left(f^{n}(z), f^{n}(x)\right)=r_{n}$ (otherwise we could extend $V$ and increase $r_{n}$ ). Hence there exists a point $z \in \partial V$, a critical point $c_{n}(x)=c_{n}$ and a number $m_{n}<n$ such that $f^{m_{n}}(z)=c_{n}$ and $d\left(f^{n}(z), f^{n}(x)\right)=r_{n}$. Let us say that $c_{n}$ generates $r_{n}$. Following [BM] (and mimicking the terminology due to Yoccoz) we call points with $r_{n} \rightarrow 0$ persistent, and points with $r_{n} \not 0$ reluctant. For example, preimages of critical points are persistent while repelling periodic non-critical points are reluctant.

Let $T$ be a metric continuum. Say that $f: T \rightarrow T$ is backward stable if for any $\delta$ there exists $\varepsilon$ such that for any continuum $K$ with $\operatorname{diam}(K) \leq \varepsilon$, any $n \geq 0$ and any component $M$ of $f^{-n}(K), \operatorname{diam}(M) \leq \delta$ (such components are called pull-backs).

Lemma 9. Let $f: T \rightarrow T$ be a backward stable piecewise local homeomorphism. Then:

(1) for any continuum $K$ there exists $\varepsilon>0$ such that $\operatorname{diam}\left(f^{n}(K)\right) \geq \varepsilon$ for all $n$;

(2) if the set $\overline{\operatorname{orb}(x)}$ does not contain critical points, then $x$ is reluctant. 
Proof. (1) Let $\delta=\operatorname{diam}(K)$. Choose $\varepsilon$ so that for any continuum $K^{\prime}$ with $\operatorname{diam}\left(K^{\prime}\right)$ $\leq \varepsilon$, any $n \geq 0$ and any component $M$ of $f^{-n}\left(K^{\prime}\right), \operatorname{diam}(M) \leq \delta / 2$. Then $\operatorname{diam}\left(f^{n}(K)\right)>\varepsilon$ for any $n$ since otherwise we take the component $M$ of $f^{-n}\left(f^{n}(K)\right)$ containing $K$ and get a contradiction because $M \supset K$ and hence with $\operatorname{diam}(M) \geq \delta$.

(2) Let $d\left(\overline{\operatorname{orb}(x)}, C_{f}\right)=\delta$. Choose $\varepsilon$ such that if $M$ is a continuum, $\operatorname{diam}(M)<\varepsilon$, then all its pull-backs are of diameter less than $\delta$. For $f^{n}(x)$ choose its connected neighborhood $V$ of diameter less than $\varepsilon$ so that its boundary is sufficiently far away from $f^{n}(x)$. Then $f^{n}$ restricted on the pull-back of $V$ containing $x$ is a local homeomorphism as desired.

Fix a locally connected unshielded plane continuum $T$ and consider a class $\mathcal{A}_{T}$ of continuous backward stable maps $f: T \rightarrow T$ of $T$ into itself such that $f$ is a local homeomorphism at all points of $T$ except of a finite set of points called critical. Now we prove Theorem 10 describing orbits of reluctant vertices under maps from $\mathcal{A}_{T}$.

Theorem 10. Let $f: T \rightarrow T, f \in \mathcal{A}_{T}$, be a map of a locally connected unshielded continuum $T$. If a local vertex of $T$ is reluctant, then it is preperiodic.

Proof. Suppose that $x$ is a reluctant local vertex. Then there exist $\varepsilon>0$, a sequence of integers $n_{k} \rightarrow \infty$ and a sequence of connected neighborhoods $U_{k}$ of $f^{n_{k}}(x)$ such that the minimal distance $d\left(f^{n_{k}}(x), \partial U_{k}\right)$ is greater than $\varepsilon$ and components $V_{k}$ of preimages $f^{-n_{k}}\left(U_{k}\right)$ containing $x$ are such that $f^{n_{k}} \mid V_{k}$ is a local homeomorphism.

Choose a triod $Y=\left[y_{1}, x\right] \cup\left[y_{2}, x\right] \cup\left[y_{3}, x\right]=Y_{1} \cup Y_{2} \cup Y_{3}$ (it is possible since $x$ is a local vertex) and consider a few cases. The simplest is when for infinitely many $k$ the components $B_{1}^{k}, B_{2}^{k}$ and $B_{3}^{k}$ of $V_{k} \backslash\{x\}$ covering small semi-neighborhoods of $x$ on $Y_{1}, Y_{2}$ and $Y_{3}$, are distinct. Fix one such $k$ and denote the components of $B_{i}^{k} \cap Y_{i}$ by $\left[z_{i}, x\right), 1 \leq i \leq 3$. A point $z_{i}$ may well coincide with $y_{i}$, but if not then $z_{i}$ must come from the boundary of $V_{k}$. By Lemma $8(3)$, sets $f^{n_{k}}\left(z_{1}, x\right), f^{n_{k}}\left(z_{2}, x\right), f^{n_{k}}\left(z_{3}, x\right)$ are pairwise disjoint. Let us show that they have bounded away from zero diameters. Indeed, if $z_{i}=y_{i}$ this holds by Lemma 9(1) (which implies that the diameters of the images of $Y_{i}$ are bounded away from zero), and if $z_{i} \neq y_{i}$, this follows by Lemma 7 (which yields that $f^{n_{k}}\left(z_{i}\right) \in \partial U_{k}$ and $d\left(f^{n_{k}}(x), \partial U_{k}\right) \geq \varepsilon$ ). This means that $f^{n_{k}}(x)$ are locally $\varepsilon^{\prime}$-essential vertices for some $\varepsilon^{\prime}>0$, and so by Lemma 6 the point $x$ is preperiodic.

Now assume that for all $k$, small semi-neighborhoods of $x$ on $Y_{1}, Y_{2}$ are contained in one component $C_{k}$ of $V_{k} \backslash\{x\}$. Since $C_{k}$ is arcwise connected by Theorem 4 , there is an arc $\left[u_{1}, u_{2}\right] \subset C_{k}$ with $u_{1} \in Y_{1} \backslash\{x\}, u_{2} \in Y_{2} \backslash\{x\}$ and $\left(u_{1}, u_{2}\right) \cap\left(Y_{1} \cup Y_{2}\right)=\emptyset$. Since $T$ is unshielded, no two points on $Y_{1}, Y_{2}$ which are closer to $x$ are connected by a similar arc. Moreover, $S=\left[u_{1}, x\right] \cup\left[x, u_{2}\right] \cup\left[u_{2}, u_{1}\right]$ is a simple closed curve, and since by the assumptions $x, u_{1}, u_{2} \in V_{k}$ then (again because $T$ is unshielded) $S \subset V_{k}$ for any $k$. By Lemma 9(1), the diameter of $f^{n_{k}}(S)$ is greater than some $\varepsilon^{\prime}$ for all $k$, and by Lemma $8(2)$ the set $f^{n_{k}}(S)$ is a simple closed curve. Hence we get two $\operatorname{arcs} I_{k}^{\prime}, I_{k}^{\prime \prime}$ in $f^{n_{k}}(S)$ of diameter $\varepsilon^{\prime} / 3$ which intersect only over their common endpoint $f^{n_{k}}(x)$.

Now, since $T$ is unshielded, a small semi-neighborhood of $x$ in $Y_{3}$ is not contained in $C_{k}$, and so there is a point $z_{3}^{k} \in Y_{3}$ such that $\left[z_{3}^{k}, x\right)$ is contained in a component of $V_{k} \backslash\{x\}$ distinct from $C_{k}$ and either $z_{3}^{k} \in \partial V_{k}$ or $z_{3}^{k}=y_{3}$. As before, this implies that the set $f^{n_{k}}\left[z_{3}^{k}, x\right)$ has a bounded away from zero diameter and is disjoint from $I_{k}^{\prime}, I_{k}^{\prime \prime}$, and again as before this implies that $x$ is preperiodic. 
Let us prove Theorem 11 which deals with the behavior of persistent vertices of a locally connected unshielded continuum $T$ under a map $f \in \mathcal{A}_{T}$. Observe that the ideas we use in the proof are quite similar to the ones used in [BM] where we deal with a more general situation under weaker than backward stability assumptions about the map.

Theorem 11 (cf. $[\mathrm{BM}]$ ). Let $f: T \rightarrow T, f \in \mathcal{A}_{T}$ be a map of a locally connected unshielded continuum $T$. If a point $x$ of $T$ is persistent, then either it is precritical or its limit set coincides with the limit set of a recurrent critical point $c(x)$ depending on $x$.

Proof. Let $x$ be a non-precritical persistent point. For every $n$ find a connected neighborhood $V$ of $x$, a connected neighborhood $U$ of $f^{n}(x)$, a critical point $c_{n}$ and a number $m_{n}$ such that $f^{n}(V)=U, f^{n} \mid V$ is a local homeomorphism, $c_{n} \in \partial f^{m_{n}}(V)$, $f^{n-m_{n}}\left(c_{n}\right) \in \partial U$ and $d\left(f^{n-m_{n}}\left(c_{n}\right), f^{n}(x)\right)=r_{n}$. Let us show that then the maximum of distances $d\left(c_{n}, f^{m_{n}}(x)\right), d\left(f\left(c_{n}\right), f^{m_{n}+1}(x)\right), \ldots, d\left(f^{n-m_{n}}\left(c_{n}\right), f^{n}(x)\right)$ converges to 0 as $n \rightarrow \infty$.

Indeed, given $\varepsilon^{\prime}>0$ choose $\varepsilon$ so that for any continuum $K \subset T$ with $\operatorname{diam}(K) \leq$ $\varepsilon$, the diameter of any component of $f^{-l}(K)$ is less than $\varepsilon^{\prime}$, for all $l \geq 0$. Now, cover $T$ with finitely many open connected neighborhoods of diameter less than $\varepsilon$ (it is possible since $T$ is locally connected). Let $\delta_{\varepsilon}$ be the Lebesgue number for this cover. This means if $r_{n}<\delta_{\varepsilon}$, then for the point $f^{n}(x) \in T$ there exists a connected neighborhood from our cover $U$ of diameter less than $\varepsilon$ such that the ball of radius $r_{n}$ centered at $f^{n}(x)$ is contained in $U$. In particular, $f^{n}(x)$ and $f^{n-m_{n}}\left(c_{n}\right)$ belong to the same connected neighborhood $U$ of diameter less than $\varepsilon$. By the choice of $\varepsilon$ this in turn implies that sets $f^{m_{n}}(\bar{V}), f^{m_{n}-1}(\bar{V}), \ldots, f^{n}(\bar{V})=$ $\bar{U}$ have diameters at most $\varepsilon^{\prime}$. Since $c_{n}, f^{m_{n}}(x) \in f^{m_{n}}(\bar{V}) ; f\left(c_{n}\right), f^{m_{n}+1}(x) \in$ $f^{m_{n}+1}(V) ; \ldots ; f^{n-m_{n}}\left(c_{n}\right), f^{n}(x) \in U$, we can easily see that the maximum of distances $d\left(c_{n}, f^{m_{n}}(x)\right), d\left(f\left(c_{n}\right), f^{m_{n}+1}(x)\right), \ldots, d\left(f^{n-m_{n}}\left(c_{n}\right), f^{n}(x)\right)$ is less than $\varepsilon^{\prime}$ which proves that, in fact, this maximum converges to 0 as $n \rightarrow \infty$.

In short, this means the following. Let $C$ be a finite collection of critical points of $f$. For any $n$ we choose a number $m_{n}$ and a point $c_{n} \in C$ such that the orbit segment $c_{n}, f\left(c_{n}\right), \ldots, f^{n-m_{n}}\left(c_{n}\right)$ approximates the orbit segment $f^{m_{n}}(x), \ldots, f^{n}(x)$ with the error of approximation $s_{n}$ (meaning that the distance $d\left(f^{i}\left(c_{n}\right), f^{m_{n}+i}(x)\right), 0 \leq$ $i \leq n-m_{n}$, is at most $\left.s_{n}\right)$ and $s_{n} \rightarrow 0$ as $n \rightarrow \infty$.

Let us show that this implies that there exists a recurrent point $t \in C$ such that $\omega(t)=\omega(x)$. First of all, let $Q$ be the set of those points $q \in C$ which appear as points $c_{n}$ infinitely many times. Then the fact that $s_{n} \rightarrow 0$ implies that $Q \subset \omega(x)$. Indeed, if $q \in Q$, then $d\left(f^{m_{n_{k}}}(x), q\right) \leq s_{n_{k}} \rightarrow 0$ along a sequence $n_{k} \rightarrow \infty$. Moreover, since $x$ by the assumption is never mapped into a critical point, then $m_{n_{k}} \rightarrow \infty$ as well. Hence $q \in \omega(x)$. Therefore $\omega(Q)=\bigcup_{q \in Q} \omega(q) \subset \omega(x)$.

On the other hand, $d\left(f^{n}(x), f^{n-m_{n}}\left(c_{n}\right)\right) \leq s_{n} \rightarrow 0$ where for big $n$ we may assume that $c_{n} \in Q$. Hence $\omega(x) \subset \omega(Q) \cup\left(\cup_{q \in Q}\right.$ orb $\left.q\right)$. Let us show that in fact this implies that $\omega(x) \subset \omega(Q)$. Indeed, otherwise there exist points $y \in \omega(x) \backslash \omega(Q)$ which then must belong to forward orbits of points of $Q$. Denote the set of all such points of $Q$ by $Q^{\prime}$; clearly, $Q^{\prime}=Q \backslash \omega(Q)$ and all points of $\omega(x) \backslash \omega(Q)$ are forward images of points of $Q^{\prime}$. Now, every point $y \in \omega(x) \backslash \omega(Q)$ has at least one preimage in $\omega(x)$ (because $f \mid \omega(x)$ is surjective) and this preimage cannot belong to $\omega(Q)$ (since $\omega(Q)$ is forward invariant and $y \notin \omega(Q)$ to begin with). Hence for every $q^{\prime} \in Q^{\prime}$ there exists $q^{\prime \prime} \in \omega(x) \backslash \omega(Q)$ such that $f\left(q^{\prime \prime}\right)=q^{\prime}$. The point $q^{\prime \prime}$ in 
turn is a forward image of some point of $Q^{\prime}$ under some iterate of $f$. Thus we can pull back the point $q^{\prime \prime}$ along this orbit and then repeat the argument. Since there are only finitely many points in the set $Q^{\prime}$, at some moment the same point from $Q^{\prime}$ will have to appear twice which means that this is a periodic point. Yet this contradicts the fact that $Q^{\prime}=Q \backslash \omega(Q)$ and shows that $\omega(x)=\omega(Q)$.

Let us show that if $Q^{\prime \prime}$ is the set of all recurrent points of $Q$, then still $\omega(x)=$ $\omega\left(Q^{\prime \prime}\right)$. Indeed, $Q \subset \omega(x)=\omega(Q)$. Hence if $q \in Q$ is not recurrent, then there is a point $\hat{q} \neq q, \hat{q} \in Q$, such that $q \in \omega(\hat{q})$. Replace the set $Q$ by the set $\hat{Q}=Q \backslash\{q\}$, then again $\hat{Q} \subset \omega(x)=\omega(\hat{Q})$ and the argument can be repeated. After finitely many steps we get the set $Q^{\prime \prime}$ for which still $\omega(x)=\omega\left(Q^{\prime \prime}\right)$.

Now, let $\tilde{Q}$ be the set of all points $\tilde{q} \in Q$ whose limit sets are maximal by inclusion among limit sets of points of $Q$. Let us show that all points of $\tilde{Q}$ are recurrent. If $z \in \tilde{Q}$ is not recurrent, then the fact that $Q \subset \omega(x)=\omega(Q)$ implies that there exists a point $z^{\prime} \in Q$ such that $z \in \omega\left(z^{\prime}\right)$ and hence $\omega(z) \subsetneq \omega\left(z^{\prime}\right)$, a contradiction to the maximality of $\omega(z)$. So, $\tilde{Q} \subset Q^{\prime \prime}$ and clearly $\omega(x)=\omega\left(Q^{\prime \prime}\right)=\omega(\tilde{Q})$.

Let us show that there exists a point $t \in \tilde{Q}$ such that $\omega(x)=\omega(t)$. By way of contradiction assume that there is no such point in $\tilde{Q}$. To continue the argument we need to make a few choices of constants and points. Choose $\gamma>0$ so that for any two points $y, z \in Q$ if $y \notin \omega(z)$, then $d(y, \omega(z))>2 \gamma$ (this is possible since $Q$ is finite). Then choose $\delta<\gamma$ so that for any two points $u, v$ the fact that $d(u, v)<\delta$ implies $d(f(u), f(v))<\gamma$ which is possible by continuity. Choose $N$ so big that $s_{n}<\delta$ for $n>N$. Choose $n>N$ such that $c_{n}=c \in \tilde{Q}$. Since $\omega\left(c_{n}\right) \neq \omega(x)$ by the assumption, then there exists the first moment $r>n$ such that $d\left(f^{r}(x), \omega(c)\right)>\delta$ (by the choice of $\gamma$ there are points in $\omega(x)$ which are more than $\gamma$-distant from $\omega(c)$, e.g. those points of $\tilde{Q}$ which do not belong to $\omega(c))$.

Consider the point $c_{r}$. Let us show that $c \in \omega\left(c_{r}\right)$ is impossible. Indeed, suppose that $c \in \omega\left(c_{r}\right)$. Then by the maximality of $\omega(c)$ we see that $\omega\left(c_{r}\right)$ is also maximal (so that $c_{r} \in \tilde{Q}$ ) and $\omega\left(c_{r}\right)=\omega(c)$. As proven above, $c_{r}$ is recurrent and since $d\left(f^{r}(x), f^{r-m_{r}}\left(c_{r}\right)\right) \leq s_{r} \leq \delta$, we see that $d\left(f^{r}(x), \omega(c)\right) \leq \delta$, a contradiction. So, $c \notin \omega\left(c_{r}\right)$. Vice versa, let us show that $c_{r} \notin \omega(c)$. Indeed, otherwise $d\left(f^{r}(x), f^{r-m_{r}}\left(c_{r}\right)\right) \leq s_{n} \leq \delta$ implies that again $d\left(f^{r}(x), \omega(c)\right) \leq \delta$, a contradiction.

Now, let us show that $m_{r}<r$. Indeed, otherwise $d\left(f^{r}(x), c_{r}\right) \leq s_{r} \leq \delta$. On the other hand, $d\left(f^{r-1}(x), \omega(c)\right) \leq \delta$ by the choice of $r$. Thus $d\left(f^{r}(x), \omega(c)\right) \leq \gamma$ by the choice of $\delta$. Together with $d\left(f^{r}(x), c_{r}\right) \leq \delta$ this implies that $d\left(c_{r}, \omega(c)\right) \leq \delta+\gamma<2 \gamma$ and hence $c_{r} \in \omega(c)$ by the choice of $\gamma$, a contradiction. So, $m_{r}<r$.

By the construction $d\left(f^{m_{r}}(x), c_{r}\right) \leq s_{r} \leq \delta$. Let us compare $m_{r}$ with $m_{n}$ and show by considering a few cases that all possibilities lead to a contradiction. First suppose that $m_{r} \geq m_{n}$. Then $d\left(f^{m_{r}}(x), \omega(c)\right) \leq \delta$ which together with $d\left(f^{m_{r}}(x), c_{r}\right) \leq \delta$ implies that $d\left(c_{r}, \omega(c)\right) \leq 2 \delta<2 \gamma$ implying as above that $c_{r} \in$ $\omega(c)$, a contradiction. Now, suppose that $m_{r}<m_{n}$. Then $d\left(f^{m_{n}-m_{r}}\left(c_{r}\right), f^{m_{n}}(x)\right)$ $\leq s_{r} \leq \delta$ and $d\left(c, f^{m_{n}}(x)\right) \leq s_{n} \leq \delta$ imply that $d\left(f^{m_{n}-m_{r}}\left(c_{r}\right), c\right)<2 \gamma$ and hence by the choice of $\gamma$ we have $c \in \omega\left(c_{r}\right)$, again the same contradiction. So, we show that the assumption that $\omega(c) \neq \omega(x)$ implies a contradiction which completes the proof of the fact that $\omega(c)=\omega(x)$.

To apply Theorems 10 and 11 we need Theorem 12 obtained in its final form in $[\mathrm{BO}$ ] (see also $\mathrm{L}$ for polynomials with one critical point whose Julia sets are locally connected continua non-separating the plane, [BL2] for polynomials with 
any number of critical points whose Julia sets are locally connected continua nonseparating the plane). To state Theorem 12 we need some information about closed invariant laminations (for the definitions see [Do], $[\mathrm{McM}],[\mathrm{Th}]$ ). Namely, if $\sim$ are such laminations, then we define an extension $\simeq$ of $\sim$ onto $\widehat{\mathbb{C}} \backslash \mathbb{D}$ by declaring that a point in $\mathbb{C} \backslash \overline{\mathbb{D}}$ is equivalent only to itself. Let $p: \mathbb{C} \backslash \mathbb{D} \rightarrow(\mathbb{C} \backslash \mathbb{D}) / \simeq$ be the factor map and denote $p\left(S^{1}\right)$ by $J$. Then $J$ is a locally connected unshielded continuum, and since the map $\sigma: S^{1} \rightarrow S^{1}$ defined by $\sigma(z)=z^{d}$ acts on $S^{1}$ and the relation $\sim$ is $\sigma$-invariant, we can consider a factor map $f: J \rightarrow J$. Notice, that the notions of a vertex and a critical point make sense for the map $f$ and that there are examples of non-polynomial laminations (see BL1]).

Theorem $12([\mathrm{BO}])$. Suppose that one of the assumptions below holds:

(1) $\sim$ is an invariant lamination and $f: J \rightarrow J$ is the corresponding factor map;

(2) $f$ is a polynomial such that its Julia set $J$ is locally connected.

Then $f \mid J$ is backward stable.

Theorems 10, 11 and 12 imply Theorem 13 which in turn implies our Main Theorem.

Theorem 13. Suppose that one of the assumptions below holds:

(1) $\sim$ is an invariant lamination and $f: J \rightarrow J$ is the corresponding factor map;

(2) $f$ is a polynomial, $J$ is its Julia set, and $J$ is locally connected.

Then if $x$ is a non-preperiodic non-precritical vertex of $J$, then $\omega(x)$ coincides with $\omega(c(x))$ for a recurrent critical point $c(x)$ depending on $x$.

\section{REFERENCES}

[BL1] A. Blokh, G. Levin, An inequality for laminations, Julia sets and "growing trees", Erg. Th. and Dyn. Syst. 22 (2002), 63-97.

[BL2] Growing trees, laminations and the dynamics on the Julia set, IHES Preprint IHES/M/99/77 (1999).

[BM] A. Blokh, M. Misiurewicz, Attractors for graph critical rational functions, Trans. Amer. Math. Soc. (to appear).

[BO] A. Blokh, l. Oversteegen, Backward stability for polynomial maps with locally connected Julia sets, Preprint, 2000.

[BH] B. Branner, J. Hubbard, The iteration of cubic polynomials. Part II: Patterns and parapatterns., Act Math. 169 (1992), 229-325. MR 94d:30044

[CJY] L. Carleson, P. Jones, J.-C. Yoccoz, Julia and John, Bol. Soc. Brasil. Mat. 25 (1994), 1-30. MR 95d:30040

[CL] E. F. Collingwood, A. J. Lohwater, The Theory of Cluster Sets, Cambridge University Press, Cambridge, 1966. MR 38:325

[Do] A. Douady, Descriptions of compact sets in $\mathbb{C}$, Topological methods in modern mathematics, Publish or Perish, 1993, pp. 429-465. MR 94g:58185

[DH] A. Douady and J. H. Hubbard, Étude dynamique des polynômes complexes I, II, Publication Mathematiques d'Orsay, vol. 84-2, 1984; vol. 85-04, 1985. MR 87f:58072a MR 87f:58072b

[F] P. Fatou, Sur les equations fonctionnelles, Bull. Soc. Math. France 47 (1919), 161-271; vol. 48, 1920, pp. 33-94, 208-314.

$[\mathrm{H}] \quad$ J. H. Hubbard, Local connectivity of Julia sets and bifurcation loci: three theorems of J.C. Yoccoz, Topological methods in modern mathematics, Publish or Perish, 1993, pp. 467511. MR 94c:58172

[J] G. Julia, Memoire sur l'iteration des fonctions rationelles, J. Math. Pure Appl. 8 (1919), 47-245.

[Ki] J. Kiwi, Rational rays and critical portraits of complex polynomials, SUNY at Stony Brook and IMS Preprint \#1997/15. 
[Kur] C. Kuratowski, Topologie, vol. 2, Warszawa-Wrocław, 1950. MR 12:517a

[L] G. Levin, On backward stability of holomorphic dynamical systems, Fundamenta Mathematicae 158 (1998), 97-107. MR 99j:58171

$[\mathrm{McM}]$ C. T. McMullen, Complex dynamics and renormalization, Annals of Mathematical Studies, vol. 135, Princeton University Press, Princeton, NJ, 1994. MR 96b:58097

[Mi] J. Milnor, Dynamics in One Complex Variable: Introductory Lectures, SUNY at Stony Brook and IMS Preprint \#1990/5.

[Po] A. Poirier, On post critically finite polynomials. Part two: Hubbard trees, SUNY at Stony Brook and IMS Preprint \#1993/7.

[Sul] D. Sullivan, Conformal dynamical systems, in: Geometric Dynamics, Lecture Notes in Math., vol. 1007, Springer Verlag, NY, 1983, pp. 725-752. MR 85m:58112

[Th] W. Thurston, The combinatorics of iterated rational maps, Preprint, 1985.

Department of Mathematics, University of Alabama in Birmingham, University Station, Birmingham, Alabama 35294-2060

E-mail address: ablokh@math.uab.edu

Institute of Mathematics, Hebrew University, Givat Ram, 91904 Jerusalem, Israel

E-mail address: levin@math.huji.ac.il 